CERN PPE/93-111

5 July 1993

\title{
Search for a non-minimal Higgs boson produced in the reaction $\mathrm{e}^{+} \mathbf{e}^{-} \rightarrow \mathrm{hZ}^{*}$
}

\author{
The ALEPH Collaboration*)
}

\begin{abstract}
A data sample corresponding to 1.23 million hadronic $\mathrm{Z}$ decays collected by the ALEPH detector at LEP has been searched for signals of the production of a non-minimal CP-even Higgs boson $h$ in the reaction $\mathrm{e}^{+} \mathrm{e}^{-} \rightarrow \mathrm{hZ}^{*}$. The $\mathrm{h}$ decay modes considered were: those of the minimal standard model Higgs boson, with modified branching ratios; decays into a pair of CP-odd Higgs bosons A; and decays into invisible final states. Only one event was found, a very acoplanar $\mathrm{e}^{+} \mathrm{e}^{-}$pair which could originate from the standard model background process $\mathrm{e}^{+} \mathrm{e}^{-} \rightarrow \mathrm{e}^{+} \mathrm{e}^{-} \nu \bar{\nu}$. Upper limits for the cross-section of the reaction $\mathrm{e}^{+} \mathrm{e}^{-} \rightarrow \mathrm{hZ}^{*}$ have been derived as a function of $m_{\mathrm{h}}$, the mass of the Higgs boson $\mathrm{h}$. In the case of invisible decays, the $95 \%$ CL lower limit on $m_{\mathrm{h}}$ is $65 \mathrm{GeV} / c^{2}$ for a production cross-section equal to that of a minimal standard model Higgs boson. When combined with previous ALEPH results on the reaction $\mathrm{e}^{+} \mathrm{e}^{-} \rightarrow \mathrm{hA}$, these cross-section upper limits exclude a domain in the $\left(m_{\mathrm{h}}, m_{\mathrm{A}}\right)$ plane of the MSSM such that, if invisible $\mathrm{h}$ and $\mathrm{A}$ decays can be neglected, $95 \% \mathrm{CL}$ lower limits of 44 and $21 \mathrm{GeV} / c^{2}$ result for $m_{\mathrm{h}}$ and $m_{\mathrm{A}}$, respectively, independent of the other parameters of the model.
\end{abstract}

(Submitted to Physics Letters B)

*) See next pages for the list of authors. 



\section{The ALEPH Collaboration}

D. Buskulic, I. De Bonis, D. Decamp, P. Ghez, C. Goy, J.-P. Lees, M.-N. Minard, B. Pietrzyk Laboratoire de Physique des Particules (LAPP), IN ${ }^{2} P^{3}$-CNRS, 74019 Annecy-le-Vieux Cedex, France

F. Ariztizabal, P. Comas, J.M. Crespo, M. Delfino, I. Efthymiopoulos, E. Fernandez, M. Fernandez-Bosman,

V. Gaitan, Ll. Garrido, T. Mattison, A. Pacheco, C. Padilla, A. Pascual Institut de Fisica d'Altes Energies, Universitat Autonoma de Barcelona, 08193 Bellaterra (Barcelona), $\operatorname{Spain}^{7}$

D. Creanza, M. de Palma, A. Farilla, G. Iaselli, G. Maggi, S. Natali, S. Nuzzo, M. Quattromini, A. Ranieri, G. Raso, F. Romano, F. Ruggieri, G. Selvaggi, L. Silvestris, P. Tempesta, G. Zito INFN Sezione di Bari e Dipartimento di Fisica dell' Università, 70126 Bari, Italy

Y. Chai, H. Hu, D. Huang, X. Huang, J. Lin, T. Wang, Y. Xie, D. Xu, R. Xu, J. Zhang, L. Zhang, W. Zhao Institute of High-Energy Physics, Academia Sinica, Beijing, The People's Republic of China ${ }^{8}$

E. Blucher, ${ }^{22}$ G. Bonvicini, J. Boudreau, D. Casper, H. Drevermann, R.W. Forty, G. Ganis, C. Gay, R. Hagelberg, J. Harvey, J. Hilgart, ${ }^{31}$ R. Jacobsen, B. Jost, J. Knobloch, I. Lehraus, T. Lohse, ${ }^{27}$ M. Maggi, C. Markou, M. Martinez, P. Mato, H. Meinhard, A. Minten, A. Miotto, R. Miquel, H.-G. Moser, P. Palazzi, J.R. Pater, J.A. Perlas, J.-F. Pusztaszeri, F. Ranjard, G. Redlinger, ${ }^{23}$ L. Rolandi, J. Rothberg, ${ }^{2}$ T. Ruan, M. Saich, D. Schlatter, M. Schmelling, F. Sefkow, ${ }^{6}$ W. Tejessy, I.R. Tomalin, R. Veenhof, H. Wachsmuth, S. Wasserbaech, ${ }^{2}$ W. Wiedenmann, T. Wildish, W. Witzeling, J. Wotschack European Laboratory for Particle Physics (CERN), 1211 Geneva 23, Switzerland

Z. Ajaltouni, F. Badaud, M. Bardadin-Otwinowska, R. El Fellous, A. Falvard, P. Gay, C. Guicheney, P. Henrard, J. Jousset, B. Michel, J-C. Montret, D. Pallin, P. Perret, F. Podlyski, J. Proriol, F. Prulhière, F. Saadi Laboratoire de Physique Corpusculaire, Université Blaise Pascal, IN ${ }^{2} P^{3}$-CNRS, Clermont-Ferrand, 63177 Aubière, France

T. Fearnley, J.B. Hansen, J.D. Hansen, J.R. Hansen, ${ }^{1}$ P.H. Hansen, R. Møllerud, B.S. Nilsson ${ }^{1}$ Niels Bohr Institute, 2100 Copenhagen, Denmark ${ }^{9}$

A. Kyriakis, E. Simopoulou, I. Siotis, A. Vayaki, K. Zachariadou Nuclear Research Center Demokritos (NRCD), Athens, Greece

J. Badier, A. Blondel, G. Bonneaud, J.C. Brient, G. Fouque, S. Orteu, A. Rougé, M. Rumpf, R. Tanaka, M. Verderi, H. Videau Laboratoire de Physique Nucléaire et des Hautes Energies, Ecole Polytechnique, IN ${ }^{2} P^{3}{ }_{-}$CNRS, 91128 Palaiseau Cedex, France

D.J. Candlin, M.I. Parsons, E. Veitch Department of Physics, University of Edinburgh, Edinburgh EH9 3JZ, United Kingdom ${ }^{10}$

E. Focardi, L. Moneta, G. Parrini Dipartimento di Fisica, Università di Firenze, INFN Sezione di Firenze, 50125 Firenze, Italy

M. Corden, C. Georgiopoulos, M. Ikeda, D. Levinthal ${ }^{15}$ Supercomputer Computations Research Institute and Dept. of Physics, Florida State University, Tallahassee, FL 32306, USA $12,13,14$

A. Antonelli, R. Baldini, G. Bencivenni, G. Bologna, ${ }^{4}$ F. Bossi, P. Campana, G. Capon, F. Cerutti, V. Chiarella, B. D’Ettorre-Piazzoli, ${ }^{24}$ G. Felici, P. Laurelli, G. Mannocchi, ${ }^{5}$ F. Murtas, G.P. Murtas, L. Passalacqua, M. PepeAltarelli, P. Picchi ${ }^{4}$ Laboratori Nazionali dell'INFN (LNF-INFN), 00044 Frascati, Italy 
P. Colrain, I. ten Have, J.G. Lynch, W. Maitland, W.T. Morton, C. Raine, P. Reeves, J.M. Scarr, K. Smith, M.G. Smith, A.S. Thompson, R.M. Turnbull

Department of Physics and Astronomy, University of Glasgow, Glasgow G12 8QQ, United Kingdom ${ }^{10}$

B. Brandl, O. Braun, C. Geweniger, P. Hanke, V. Hepp, E.E. Kluge, Y. Maumary, A. Putzer, B. Rensch, A. Stahl, K. Tittel, M. Wunsch

Institut für Hochenergiephysik, Universität Heidelberg, 6900 Heidelberg, Fed. Rep. of Germany ${ }^{16}$

R. Beuselinck, D.M. Binnie, W. Cameron, M. Cattaneo, D.J. Colling, P.J. Dornan, A.M. Greene, J.F. Hassard, N.M. Lieske, ${ }^{29}$ A. Moutoussi, J. Nash, S. Patton, D.G. Payne, M.J. Phillips, G. San Martin, J.K. Sedgbeer, A.G. Wright

Department of Physics, Imperial College, London SW7 2BZ, United Kingdom ${ }^{10}$

P. Girtler, D. Kuhn, G. Rudolph, R. Vogl

Institut für Experimentalphysik, Universität Innsbruck, 6020 Innsbruck, Austria ${ }^{18}$

C.K. Bowdery, T.J. Brodbeck, A.J. Finch, F. Foster, G. Hughes, D. Jackson, N.R. Keemer, M. Nuttall, A. Patel, T. Sloan, S.W. Snow, E.P. Whelan

Department of Physics, University of Lancaster, Lancaster LA1 4YB, United Kingdom ${ }^{10}$

K. Kleinknecht, J. Raab, B. Renk, H.-G. Sander, H. Schmidt, F. Steeg, S.M. Walther, R. Wanke, B. Wolf Institut für Physik, Universität Mainz, 6500 Mainz, Fed. Rep. of Germany ${ }^{16}$

A.M. Bencheikh, C. Benchouk, A. Bonissent, J. Carr, P. Coyle, J. Drinkard, ${ }^{3}$ F. Etienne, D. Nicod, S. Papalexiou, P. Payre, L. Roos, D. Rousseau, P. Schwemling, M. Talby

Centre de Physique des Particules, Faculté des Sciences de Luminy, IN ${ }^{2} P^{3}$-CNRS, 13288 Marseille,

France

S. Adlung, R. Assmann, C. Bauer, W. Blum, D. Brown, P. Cattaneo, ${ }^{26}$ B. Dehning, H. Dietl, F. Dydak, ${ }^{21}$ M. Frank, A.W. Halley, K. Jakobs, J. Lauber, G. Lütjens, G. Lutz, W. Männer, R. Richter, J. Schröder, A.S. Schwarz, R. Settles, H. Seywerd, U. Stierlin, U. Stiegler, R. St. Denis, G. Wolf Max-Planck-Institut für Physik, Werner-Heisenberg-Institut, 8000 München, Fed. Rep. of Germany ${ }^{16}$

R. Alemany, J. Boucrot, ${ }^{1}$ O. Callot, A. Cordier, M. Davier, L. Duflot, J.-F. Grivaz, Ph. Heusse, D.E. Jaffe, P. Janot, D.W. Kim ${ }^{19}$ F. Le Diberder, J. Lefrançois, A.-M. Lutz, M.-H. Schune, J.-J. Veillet, I. Videau, Z. Zhang Laboratoire de l'Accélérateur Linéaire, Université de Paris-Sud, IN ${ }^{2} P^{3}$ CNRS, 91405 Orsay Cedex, France

D. Abbaneo, G. Bagliesi, G. Batignani, U. Bottigli, C. Bozzi, G. Calderini, M. Carpinelli, M.A. Ciocci, R. Dell'Orso, I. Ferrante, F. Fidecaro, L. Foà, F. Forti, A. Giassi, M.A. Giorgi, A. Gregorio, F. Ligabue, A. Lusiani, E.B. Mannelli, P.S. Marrocchesi, A. Messineo, F. Palla, G. Rizzo, G. Sanguinetti, P. Spagnolo, J. Steinberger, R. Tenchini, G. Tonelli, ${ }^{32}$ G. Triggiani, C. Vannini, A. Venturi, P.G. Verdini, J. Walsh

Dipartimento di Fisica dell'Università, INFN Sezione di Pisa, e Scuola Normale Superiore, 56010 Pisa, Italy

A.P. Betteridge, Y. Gao, M.G. Green, P.V. March, Ll.M. Mir, T. Medcalf, I.S. Quazi, J.A. Strong, L.R. West Department of Physics, Royal Holloway \& Bedford New College, University of London, Surrey TW20 OEX, United Kingdom ${ }^{10}$

D.R. Botterill, R.W. Clifft, T.R. Edgecock, S. Haywood, P.R. Norton, J.C. Thompson Particle Physics Dept., Rutherford Appleton Laboratory, Chilton, Didcot, Oxon OX11 OQX, United King dom ${ }^{10}$

B. Bloch-Devaux, P. Colas, H. Duarte, S. Emery, W. Kozanecki, E. Lançon, M.C. Lemaire, E. Locci, B. Marx, P. Perez, J. Rander, J.-F. Renardy, A. Rosowsky, A. Roussarie, J.-P. Schuller, J. Schwindling, D. Si Mohand, B. Vallage

Service de Physique des Particules, DAPNIA, CE-Saclay, 91191 Gif-sur-Yvette Cedex, France ${ }^{17}$ 
R.P. Johnson, A.M. Litke, G. Taylor, J. Wear

Institute for Particle Physics, University of California at Santa Cruz, Santa Cruz, CA 95064, USA ${ }^{25}$

J.G. Ashman, W. Babbage, C.N. Booth, C. Buttar, S. Cartwright, F. Combley, I. Dawson, L.F. Thompson

Department of Physics, University of Sheffield, Sheffield S3 $7 R H$, United Kingdom ${ }^{10}$

E. Barberio, A. Böhrer, S. Brandt, G. Cowan, C. Grupen, G. Lutters, F. Rivera, ${ }^{30}$ U. Schäfer, L. Smolik

Fachbereich Physik, Universität Siegen, 5900 Siegen, Fed. Rep. of Germany ${ }^{16}$

L. Bosisio, R. Della Marina, G. Giannini, B. Gobbo, F. Ragusa ${ }^{20}$

Dipartimento di Fisica, Università di Trieste e INFN Sezione di Trieste, 34127 Trieste, Italy

L. Bellantoni, W. Chen, J.S. Conway, ${ }^{28}$ Z. Feng, D.P.S. Ferguson, Y.S. Gao, J. Grahl, J.L. Harton, O.J. Hayes III, J.M. Nachtman, Y.B. Pan, Y. Saadi, M. Schmitt, I. Scott, V. Sharma, Z.H. Shi, J.D. Turk, A.M. Walsh, F.V. Weber, Sau Lan Wu, X. Wu, M. Zheng, G. Zobernig

Department of Physics, University of Wisconsin, Madison, WI 53706, USA ${ }^{11}$

\footnotetext{
${ }^{1}$ Now at CERN, PPE Division, 1211 Geneva 23 , Switzerland.

${ }^{2}$ Permanent address: University of Washington, Seattle, WA 98195, USA.

${ }^{3}$ Now at University of California, Irvine, CA 92717, USA.

${ }^{4}$ Also Istituto di Fisica Generale, Università di Torino, Torino, Italy.

${ }^{5}$ Also Istituto di Cosmo-Geofisica del C.N.R., Torino, Italy.

${ }^{6}$ Now at DESY, Hamburg, Germany.

${ }^{7}$ Supported by CICYT, Spain.

${ }^{8}$ Supported by the National Science Foundation of China.

${ }^{9}$ Supported by the Danish Natural Science Research Council.

${ }^{10}$ Supported by the UK Science and Engineering Research Council.

${ }^{11}$ Supported by the US Department of Energy, contract DE-AC02-76ER00881.

${ }^{12}$ Supported by the US Department of Energy, contract DE-FG05-87ER40319.

${ }^{13}$ Supported by the NSF, contract PHY-8451274.

${ }^{14}$ Supported by the US Department of Energy, contract DE-FC05-85ER250000.

${ }^{15}$ Supported by SLOAN fellowship, contract BR 2703.

${ }^{16}$ Supported by the Bundesministerium für Forschung und Technologie, Fed. Rep. of Germany.

${ }^{17}$ Supported by the Direction des Sciences de la Matière, C.E.A.

${ }^{18}$ Supported by Fonds zur Förderung der wissenschaftlichen Forschung, Austria.

${ }^{19}$ Supported by the Korean Science and Engineering Foundation and Ministry of Education.

${ }^{20}$ Now at Dipartimento di Fisica, Università di Milano, Milano, Italy.

${ }^{21}$ Also at CERN, PPE Division, 1211 Geneva 23, Switzerland.

${ }^{22}$ Now at University of Chicago, Chicago, IL 60637, U.S.A.

${ }^{23}$ Now at TRIUMF, Vancouver, B.C., Canada.

${ }^{24}$ Also at Università di Napoli, Dipartimento di Scienze Fisiche, Napoli, Italy.

${ }^{25}$ Supported by the US Department of Energy, grant DE-FG03-92ER40689.

${ }^{26}$ Now at Università di Pavia, Pavia, Italy.

${ }^{27}$ Now at Max-Planck-Institut f. Kernphysik, Heidelberg, Germany.

${ }^{28}$ Now at Rutgers University, Piscataway, NJ 08854, USA.

${ }^{29}$ Now at Oxford University, Oxford OX1 3RH, U.K.

${ }^{30}$ Partially supported by Colciencias, Colombia.

${ }^{31}$ Now at SSCL, Dallas 75237-3946, TX, U.S.A.

${ }^{32}$ Also at Istituto di Matematica e Fisica, Università di Sassari, Sassari, Italy.
} 



\section{Introduction}

The searches for the Higgs boson of the minimal standard model (MSM) produced in the bremsstrahlung process $\mathrm{e}^{+} \mathrm{e}^{-} \rightarrow \mathrm{HZ}^{*}$ can in principle be applied to a CP-even Higgs boson $\mathrm{h}$ of any non-minimal model, taking into account a model dependent factor, denoted $\xi^{2}$ in the following, by which the production rate is reduced.

In this letter, only those non-minimal models [1] are considered which contain Higgs fields in $S U(2)_{L}$ representations not larger than doublets. In this case, the tree-level value of $\rho$ [2] automatically remains equal to unity, as required by precision electroweak measurements. In addition, in order to avoid flavour changing neutral currents, one of the Higgs doublets, say the first one, is required to couple to down-type quarks and to charged leptons only while the second one couples to up-type quarks only. The ratio $v_{2} / v_{1}$ of the vacuum expectation values developed by the neutral components of the two Higgs doublets is denoted $\tan \beta$. Finally, additional Higgs field singlets may or may not be present.

In these models, the couplings of the Higgs boson h to down-type quarks and to charged leptons remain in the same ratios as in the MSM. In particular, this is the case for the ratio of the $h b b$ and $h \tau \tau$ couplings. On the other hand, the ratios of the hec to the $h b b$ or $\mathrm{h} \tau \tau$ couplings can be very different from their MSM values.

A well known example of such non-minimal models is the minimal supersymmetric extension of the standard model (MSSM) [1] which does not contain additional singlets and in which $\xi^{2}$ is equal to $\sin ^{2}(\beta-\alpha)$, where $\alpha$ is the mixing angle in the CP-even Higgs sector. In the MSSM, it is commonly assumed that $\tan \beta>1$, in which case the couplings of $h$ to down-type quarks (such as the b-quark) and to charged leptons (such as the $\tau$ ) are enhanced with respect to those of the MSM Higgs boson, and the couplings to uptype quarks (such as the c-quark) are reduced. The analyses presented in this letter are conducted in a framework broader than the MSSM, but the implications for this model of the results obtained are also detailed in the end.

The results of the MSM Higgs boson searches can be directly used in the context of a non-minimal model provided that the detection efficiencies are identical in the two cases. This, however, is likely not to be true if the Higgs boson decay branching ratios and, even more so, if the decay channels themselves are different. The selection criteria used by ALEPH in the search for the standard model Higgs boson are described in detail in Refs. 3 and 4 . 
With these criteria, there are a few situations in which detection efficiency differences can affect the results in a significant way:

- if, although the decay channels are the same, the branching ratios are modified in such a way that the various final state topologies contribute differently;

- if the decay channel $\mathrm{h} \rightarrow \mathrm{AA}$ is kinematically allowed (A is a CP-odd neutral Higgs boson which is not present in the MSM);

- if other decay channels not existing in the MSM are available, in particular invisible decay modes $[5,6]$.

In the first of these instances, the selection efficiencies determined in the MSM case for every decay channel $[3,4]$ can simply be reweighted in the most conservative way in order to take into account any possible differences in the decay branching ratios.

Dedicated searches were designed and applied to the data collected in 1989 and 1990 [3], taking into account the specific features of the $h \rightarrow A A$ channel [7]. The search criteria have been extended to be sensitive to the decays of the $\mathrm{A}$ boson into final states containing only neutral particles. The case of a very light $\mathrm{A}\left(m_{\mathrm{A}}<2 m_{\mu}\right)$, already investigated in detail and excluded in a previous publication [8] within the MSSM framework for any $m_{\mathrm{h}}$ and for any $\tan \beta>1$, is no longer considered here.

If other new decay modes are available, the most dramatic changes arise when the Higgs boson can decay invisibly. This may happen, for instance, in R-parity conserving supersymmetric models when the lightest neutralino $\chi$ is light enough for the decay $\mathrm{h} \rightarrow \chi \chi$ to be allowed ( $\chi$ is commonly expected to be the lightest supersymmetric particle, and therefore invisible). The search for such an invisible Higgs boson has now been performed for the first time.

The data sample used to obtain the results presented in the following corresponds to 1.23 million hadronic $\mathrm{Z}$ decays collected by the ALEPH detector at LEP from 1989 to 1992. A thorough description of the detector can be found in Ref. 9, and a brief account in Ref. 4.

In order to assess the selectivity of the various searches described in the following, large Monte Carlo samples of all standard model background processes have been used, in particular: 1.4 million $\mathrm{Z} \rightarrow \mathrm{q} \overline{\mathrm{q}}$ events; $100000 \mathrm{Z} \rightarrow \tau^{+} \tau^{-}$events, corresponding to 1.6 times the data statistics; 20000 events from the two-photon process $\gamma \gamma \rightarrow \tau^{+} \tau^{-}$and 10000 from $\gamma \gamma \rightarrow c \bar{c}$, corresponding to 1.8 and 1.4 times the recorded integrated luminosity, respectively. In addition, smaller simulated event samples, but all corresponding to at least twice the data statistics, have been analysed for the various processes $\mathrm{e}^{+} \mathrm{e}^{-} \rightarrow 1^{+} 1^{-} \overline{f f}$, with $\overline{f f}=1^{+} 1^{-}$or $q \bar{q}$. The search efficiencies for the reaction $\mathrm{e}^{+} \mathrm{e}^{-} \rightarrow \mathrm{hZ}^{*}$ have been determined using Monte Carlo samples produced with a minimal standard model Higgs boson generator, modified to accommodate non-standard Higgs decays. 


\section{Results valid when no new decay channels contribute}

For $m_{\mathrm{h}}>2 m_{\mathrm{b}}$, the configurations considered are [4] $(\mathrm{h} \rightarrow$ hadrons $)\left(\mathrm{Z}^{*} \rightarrow \nu \bar{\nu}\right)$ and, if $m_{\mathrm{h}}>40 \mathrm{GeV} / c^{2},(\mathrm{~h} \rightarrow$ hadrons $)\left(\mathrm{Z}^{*} \rightarrow \mathrm{e}^{+} \mathrm{e}^{-}\right.$or $\left.\mu^{+} \mu^{-}\right)$. With the search criteria described in detail in Ref. 4 , no candidates were found in the full data sample, leading to a mass lower limit of $58.4 \mathrm{GeV} / c^{2}$ for the MSM Higgs boson. For a non-minimal Higgs boson, the search efficiency with respect to the MSM case is essentially controlled by the value of the branching ratio of $h \rightarrow$ hadrons, as compared to that of $h \rightarrow \tau^{+} \tau^{-}$. In the models considered here, this hadronic decay branching ratio reaches its minimum value when the hec coupling vanishes, in which case it is only $1 \%$ smaller than in the MSM. The efficiency determined in the MSM Higgs boson search, displayed in Table 1 of Ref. 4, has been conservatively reduced by this amount.

For the mass range $2 m_{\mu}<m_{\mathrm{h}}<2 m_{\mathrm{b}}$, only the $\mathrm{Z}^{*} \rightarrow \nu \bar{\nu}$ decay is considered [3]. The relevant searches, i.e. those for acoplanar lepton pairs and for monojets, are described in detail in Section 4. They require, in particular, a charged particle multiplicity of at least two. One candidate event has been found, an acoplanar $\mathrm{e}^{+} \mathrm{e}^{-}$pair. The following mass ranges can be distinguished.

- $2 m_{\tau}<m_{\mathrm{h}}<2 m_{\mathrm{b}}$

The search efficiency is lower for $\mathrm{h} \rightarrow \tau^{+} \tau^{-}$than for $\mathrm{h} \rightarrow$ hadrons. Therefore, the results are derived assuming that $h$ mainly decays into $\tau^{+} \tau^{-}$. The resulting efficiency is $73 \%$ of that applying in the MSM case. Since the selected acoplanar $\mathrm{e}^{+} \mathrm{e}^{-}$pair mentioned above may result from a $\tau^{+} \tau^{-}$final state, it is kept as a candidate in this mass range.

- $2 m_{\pi}<m_{\mathrm{h}}<2 m_{\tau}$

The detection efficiency is lowest when the fraction of final states containing no charged particles is largest. Given the decay channels allowed for a CP-even Higgs boson in this mass range [10], the worst configuration is reached when h decays into $\eta \eta$, in which case half of the final states contain only neutral particles. Conservatively, the results are derived assuming that same fraction to hold over the whole mass range, except for $m_{\mathrm{h}}<2 m_{\mathrm{K}}$ where only $\mathrm{h} \rightarrow \pi \pi$ is considered, with a $\pi^{0} \pi^{0}$ fraction of one third. Here, the $\mathrm{e}^{+} \mathrm{e}^{-}$pair is not taken as a candidate because the branching ratio of $\mathrm{h} \rightarrow \mathrm{e}^{+} \mathrm{e}^{-}$is always negligible in this mass range.

- $2 m_{\mu}<m_{\mathrm{h}}<2 m_{\pi}$

The only relevant decay channel is $h \rightarrow \mu^{+} \mu^{-}$, and the search efficiency determined in the MSM case applies without modification. No events have been found.

For $m_{\mathrm{h}}<2 m_{\mu}$, the lifetime of the Higgs boson can no longer be ignored. If the lifetime is very large, the search for an invisible Higgs boson reported in Section 4 applies and, anticipating the results obtained therein, the $95 \%$ CL upper limit on $\xi^{2}$ is found to be $2.310^{-3}$. For sufficiently small lifetimes, the search for acoplanar lepton pairs described in Section 4 could be used for the configuration $\left(\mathrm{h} \rightarrow \mathrm{e}^{+} \mathrm{e}^{-}\right)\left(\mathrm{Z}^{*} \rightarrow \nu \bar{\nu}\right)$. However, since the competing $\mathrm{h} \rightarrow \gamma \gamma$ decay mode proceeds via loop diagrams, its rate is sensitive to the 
details of the model. Therefore, no general statement can be made for this mass range without a dedicated analysis.

The $95 \%$ CL upper limit on $\xi^{2}$ resulting from these studies is displayed in Fig. 1 as a function of $m_{\mathrm{h}}$. For $\xi^{2}=1$, the lower limit on $m_{\mathrm{h}}$ is $58.4 \mathrm{GeV} / c^{2}$.

\section{Results valid when the $h \rightarrow$ AA decay channel is dominant}

Here, the value of the $\mathrm{A}$ mass plays an important rôle. If $m_{\mathrm{A}} \gtrsim 2 m_{\mathrm{b}}$, the final state resulting from $\mathrm{h} \rightarrow \mathrm{AA}$ does not show any features, for instance in the charged multiplicity, which could lower the selection efficiency with respect to the MSM case. However, for the mass ranges $2 m_{\tau}<m_{\mathrm{A}} \lesssim 2 m_{\mathrm{b}}$ (in which $\mathrm{A} \rightarrow \tau^{+} \tau^{-}$may dominate over $\mathrm{A} \rightarrow \mathrm{c} \overline{\mathrm{c}}$ ) and $m_{\mathrm{A}}<2 m_{\tau}$, the final state resulting from $\mathrm{h} \rightarrow \mathrm{AA}$ may exhibit a charged multiplicity which is too low compared to the requirements [4] applied in the search for a similar mass MSM Higgs boson.

Specific searches have been designed previously [3] for the configurations $(\mathrm{h} \rightarrow \mathrm{AA}$ ) $\left(\mathrm{Z}^{*} \rightarrow \mathrm{e}^{+} \mathrm{e}^{-}\right.$or $\mu^{+} \mu^{-}$or $\left.\nu \bar{\nu}\right)$, with the resulting $\mathrm{A}$ bosons decaying into low mass and low charged multiplicity systems. The search criteria have been extended in order to be sensitive to final states in which one of the two A bosons decays into a system containing only neutral particles, which may occasionally happen in the case of an A boson mass below the $\tau^{+} \tau^{-}$and $c \bar{c}$ thresholds $\left(e . g\right.$. if $\mathrm{A} \rightarrow \eta \eta \eta$ with $\eta \rightarrow \gamma \gamma$ or $\left.3 \pi^{0}\right)$.

The selection criteria for $(\mathrm{h} \rightarrow \mathrm{AA})\left(\mathrm{Z}^{*} \rightarrow \nu \bar{\nu}\right)$ are listed below.

The total charged multiplicity must be 2,4 or 6 , including tracks possibly coming from long lived neutral particle $\left(\mathrm{V}^{0}\right)$ decays, and the total electric charge must be zero. Larger charged multiplicities are covered by the MSM Higgs boson search in the acoplanar jet topology [4]. In order to avoid energy losses in the very forward and backward directions, it is required that no energy be detected within $12^{\circ}$ of the beam axis, and that no track be reconstructed with a polar angle $\theta$ with respect to that axis such that $|\cos \theta|>0.95$. Events resulting from two photon processes are largely eliminated by the requirement that the total momentum transverse to the beam direction exceed $5 \%$ of the centre-of-mass energy.

The remaining events are divided into two hemispheres by a plane perpendicular to the thrust axis. Each hemisphere must contain no more than 4 tracks with a total electric charge equal to zero. In each hemisphere, the total energy must exceed $2 \mathrm{GeV}$ and, in order to ensure that the final state is well contained in the detector, the direction of the total momentum is required to have a polar angle $\theta$ such that $|\cos \theta|<0.9$. As in the search for acoplanar jets, the space angle between the directions of the total momenta in the two hemispheres (acollinearity angle) is required to be smaller than $165^{\circ}$, and the angle between the directions of these total momenta projected onto a plane transverse to the beam axis (acoplanarity angle) not to exceed $175^{\circ}$.

Finally, if one of the two hemispheres contains no charged particles, the total mass in each hemisphere is required to be smaller than $4 \mathrm{GeV} / c^{2}$. While this criterion largely 
eliminates the background from $\tau \tau \gamma$ final states in which one of the two hemispheres contains only a radiated photon, it does not introduce any inefficiency for the signal since an $\mathrm{A}$ boson with mass in excess of $2 m_{\tau}$ always has a negligible branching ratio into final states containing only neutral particles.

No events have been found in the data or in the standard process Monte Carlo samples. When combined with the search for the MSM Higgs boson in the configuration $(\mathrm{H} \rightarrow$ hadrons $)\left(\mathrm{Z}^{*} \rightarrow \nu \bar{\nu}\right)$, the efficiency of this search is $39 \%$ for $m_{\mathrm{h}}=60 \mathrm{GeV} / c^{2}$ and $m_{\mathrm{A}}=6 \mathrm{GeV} / c^{2}$ (with a branching ratio of $85 \%$ for $\mathrm{A} \rightarrow \tau^{+} \tau^{-}$).

The selection criteria for $(\mathrm{h} \rightarrow \mathrm{AA})\left(\mathrm{Z}^{*} \rightarrow \mathrm{e}^{+} \mathrm{e}^{-}\right.$or $\left.\mu^{+} \mu^{-}\right)$are listed below.

The total charged multiplicity must be 4 or 6 , including tracks possibly coming from $\mathrm{V}^{0}$ decays, and the total electric charge must be zero. Larger charged multiplicities are covered by the MSM Higgs boson search in the energetic lepton pair in hadronic events topology [4]. Again, no track may be reconstructed with $|\cos \theta|>0.95$, and no energy may be detected within $12^{\circ}$ of the beam axis.

The event must contain a charged particle pair satisfying the following conditions. The two particles must come from the beam crossing point and must have opposite electric charges. As in the search for energetic lepton pairs in hadronic events, both momenta are required to exceed $3 \mathrm{GeV} / c$, their scalar sum must be larger than $20 \mathrm{GeV} / c$, and the invariant mass of the pair must not be smaller than $5 \mathrm{GeV} / c^{2}$. The particles must be identified as two electrons or as two muons, at least one of them tightly, using the criteria applied in the corresponding MSM Higgs boson search [3]. Electrons belonging to an identified photon conversion are rejected.

The two lepton tracks are then removed, and the thrust axis of the rest of the event is determined. As in the search for energetic lepton pairs in hadronic events, the scalar sum of the transverse momenta of the two leptons, calculated with respect to that axis, is required to exceed $15 \mathrm{GeV} / c$. With the two leptons still removed, the event is divided into two hemispheres by a plane perpendicular to that same axis. In each hemisphere, the total electric charge is required to be equal to zero and the total energy to exceed $2 \mathrm{GeV}$. Hemispheres consisting of an identified photon conversion are rejected. In order to remove the large fraction of the background from $1^{+} 1^{-} \mathrm{ff} \gamma$ final states in which the fff or the photon direction is close to that of one of the leptons, all masses obtained by combining a lepton with the content of an hemisphere are required to be larger than $2 \mathrm{GeV} / c^{2}$.

Finally, if one of the two hemispheres contains no charged particles, the total mass in each hemisphere is again required to be smaller than $4 \mathrm{GeV} / c^{2}$.

Requiring, as in the MSM Higgs boson search, the mass recoiling against the lepton pair to be larger than $40 \mathrm{GeV} / c^{2}$, no events have been found in the data, and none in the standard process Monte Carlo samples either. When combined with the search for the MSM Higgs boson in the configuration ( $\mathrm{H} \rightarrow$ hadrons) $\left(\mathrm{Z}^{*} \rightarrow \mathrm{e}^{+} \mathrm{e}^{-}\right.$or $\left.\mu^{+} \mu^{-}\right)$, the efficiency of this search is $45 \%$ for $m_{\mathrm{h}}=60 \mathrm{GeV} / c^{2}$ and $m_{\mathrm{A}}=6 \mathrm{GeV} / c^{2}$ (with a branching ratio of $85 \%$ for $\mathrm{A} \rightarrow \tau^{+} \tau^{-}$). 
If the cut on the recoil mass is removed, two events are selected, with recoil masses of 7.5 and $12.5 \mathrm{GeV} / c^{2}$. In both cases, one of the hemispheres does not contain any charged particles. These two events can be interpreted as coming from the reactions $\mathrm{e}^{+} \mathrm{e}^{-} \rightarrow 1^{+} 1^{-} \mathrm{ff} \gamma$, with one of the hemispheres consisting of a single photon and the other of the $\overline{f f}$ system. Indeed, $\sim 2$ such events are expected to be selected in the full data sample, according to simulations performed with the FERMISV four-fermion final state generator [11] which includes initial and final state radiation. It is because of this background that the search with energetic lepton pairs has been restricted to recoil masses above $40 \mathrm{GeV} / c^{2}$.

The overall search efficiency obtained when $h \rightarrow A A$ is similar to the one achieved for a MSM Higgs boson when $m_{\mathrm{A}} \gtrsim 2 m_{\mathrm{b}}$, and lower by no more than $10 \%$, in relative value, for lower A masses. For $m_{\mathrm{A}}<2 m_{\tau}$, this is due in particular to the fact that the fraction of final states containing no charged particles in a $h \rightarrow$ AA decay never exceeds $13 \%$, a maximum which is reached, given the decay channels allowed for a CP-odd Higgs boson in this mass range [10], when both A bosons decay into $\eta \eta \eta$.

As a result, the $95 \%$ CL upper limit on $\xi^{2}$ obtained in the previous section and shown in Fig. 1 applies in the present context for $m_{\mathrm{A}} \gtrsim 2 m_{\mathrm{b}}$, and is degraded by at most $10 \%$ otherwise, in which case the lower limit on $m_{\mathrm{h}}$ is reduced to $57.8 \mathrm{GeV} / c^{2}$ for $\xi^{2}=1$.

\section{Search for an invisible Higgs boson}

The topologies associated with the production of an invisible Higgs boson $\mathrm{h}$ in the reaction $\mathrm{e}^{+} \mathrm{e}^{-} \rightarrow \mathrm{hZ}^{*}$ depend on the decay channel and on the mass of the $\mathrm{Z}^{*}$. They are:

- a purely invisible final state in the $20 \%$ of cases in which $\mathrm{Z}^{*} \rightarrow \nu \bar{\nu}$;

- an acoplanar lepton pair in the $10 \%$ of cases in which $\mathrm{Z}^{*} \rightarrow 1^{+} 1^{-}$;

- an acoplanar pair of jets or a monojet, depending whether the $\mathrm{Z}^{*}$ mass is large or small compared to its momentum, in the remaining $70 \%$ of cases in which $\mathrm{Z}^{*} \rightarrow$ hadrons.

The purely invisible final state contributes far too little to the $\mathrm{Z}$ invisible width to be useful. The search for acoplanar jets, optimized for the case of a high mass standard model Higgs boson produced in the reaction $\mathrm{e}^{+} \mathrm{e}^{-} \rightarrow \mathrm{H} \nu \bar{\nu}$ and decaying into hadrons, is described in Ref. 4. No candidate events were found. The results can be reinterpreted in the present context, with the rôles of the Higgs boson and of the $\mathrm{Z}^{*}$ simply interchanged.

The search for monojets, complemented by the search for acoplanar pairs described below, was optimized for a low mass Higgs boson produced in the same reaction. The selection criteria listed below are identical to those described in Ref. 3 .

The charged multiplicity must be at least 4. (Monojets containing only two charged particles are treated as acoplanar lepton pairs, as described below.) The events are divided into two hemispheres by a plane perpendicular to the thrust axis, and one of the two hemispheres is required to contain an energy smaller than $2 \mathrm{GeV}$ while the other hemisphere is called the monojet. In order to ensure that the final state is 
well contained in the detector, it is required that $|\cos \theta|<0.9$ for the monojet, and that no energy be measured within $12^{\circ}$ of the beam axis. A clear monojet topology is achieved by the requirement that the cone of half-opening angle $50^{\circ}$ around the direction opposite to that of the monojet contain no energy.

At this stage, most of the remaining background comes from two-photon processes and is largely reduced by the requirement that the component of the total momentum transverse to the beam direction exceed $5 \%$ of the centre-of-mass energy. To eliminate the few events surviving in the standard two-photon process Monte Carlo samples, the projected acoplanarity $\psi_{p}$ is required to be smaller than $150^{\circ}$. Here, $\psi_{p}$ is calculated as follows: all particle momenta are projected onto a plane perpendicular to the beam axis; a 2d-thrust axis is computed from these projections; the event is divided into two $2 \mathrm{~d}$-jets by a line perpendicular to that axis; $\psi_{p}$ is the angle between the directions of these two $2 \mathrm{~d}$-jets (for $2 \mathrm{~d}$-monojets, $\psi_{p}$ is set to zero).

No monojet candidate events were found in the data or in the standard process Monte Carlo samples.

The selection criteria for acoplanar lepton pairs, updated from Ref. 3, are described below.

The topology of interest consists of a pair of charged particles or $\tau$ decay products accompanied by missing energy. Events with 2 or 4 charged particle tracks and total electric charge zero are considered. Triplets of tracks with an invariant mass smaller than $1.5 \mathrm{GeV} / c^{2}$ are selected as $\tau$ candidates. If more than one triplet fulfils these conditions, only the one with the lowest mass is considered. For simplicity, the $\tau$ triplets and the remaining charged particle tracks are referred to as leptons, and only the events with two leptons are further considered. In order to avoid energy losses in the very forward and backward directions, it is required that no energy be detected within $12^{\circ}$ of the beam axis, and that $|\cos \theta|<0.95$ for each lepton.

To reduce the large $\mathrm{Z} \rightarrow 1^{+} 1^{-}$background, it is required that the angle between the two lepton directions be smaller than $165^{\circ}$. Events from $\mathrm{Z} \rightarrow 1^{+} 1^{-} \gamma$ are eliminated by a "photon veto". An event is vetoed if it contains a neutral particle with energy above $1 \mathrm{GeV}$. In order not to veto acoplanar $\tau$ pairs, neutral particles with a momentum direction closer than $10^{\circ}$ to that of one of the leptons or which form an invariant mass smaller than $2 \mathrm{GeV} / c^{2}$ with one of the leptons are not considered in the veto procedure. In order to retain efficiency for low multiplicity monojets, the photon veto is applied only if the total visible mass of the event exceeds $10 \mathrm{GeV} / c^{2}$.

The component of the vector sum of the lepton momenta transverse to the beam axis must exceed $5 \%$ of the centre-of-mass energy. The same cut is applied to the transverse component of the total visible momentum. These missing transverse momentum cuts eliminate most of the background from the two-photon processes $\gamma \gamma \rightarrow 1^{+} 1^{-}$. However, some events from $\gamma \gamma \rightarrow \tau^{+} \tau^{-}$are expected to survive because of the transverse momentum carried away by the $\tau$-decay neutrinos. To eliminate this background, the following procedure is applied: the lepton momenta are projected onto a plane perpendicular to the beam axis, and a $2 \mathrm{~d}$-thrust axis is computed from these projections; 
the scalar sum of the transverse components of the 2d-lepton momenta, measured with respect to the $2 \mathrm{~d}$-thrust axis, is required to be larger than $2 \mathrm{GeV} / c$.

This last cut is not applied if the event is monojet-like, i.e. if the $2 \mathrm{~d}$-thrust axis points between the two projected lepton momenta. In such a case, criteria designed for the monojet search are applied instead: the monojet direction must have $|\cos \theta|<0.9$, the hemisphere opposite to the monojet direction must not contain an energy exceeding $2 \mathrm{GeV}$, and no energy may be detected in the cone of half-opening angle $50^{\circ}$ around the direction opposite to that of the monojet. Finally, the monojet must not consist of an identified photon conversion.

No events have been selected in the standard process Monte Carlo samples, but a very acoplanar $\mathrm{e}^{+} \mathrm{e}^{-}$pair, shown in Fig. 2, was found in the data. The mass recoiling against the two leptons, i.e. the mass of the invisible Higgs boson candidate, is $61.3 \mathrm{GeV} / \mathrm{c}^{2}$, with a resolution of $0.6 \mathrm{GeV} / c^{2}$. This event lies far from all the selection cuts. Its salient features are an $\mathrm{e}^{+} \mathrm{e}^{-}$mass $m$ of $3.31 \pm 0.02 \mathrm{GeV} / c^{2}$ and a missing transverse momentum $p_{t}$ of $20.3 \mathrm{GeV} / c$. Within the standard model, its most likely origin is the process $\mathrm{e}^{+} \mathrm{e}^{-} \rightarrow 1^{+} 1^{-} \nu \bar{\nu}$. The expected number of such events and their kinematic characteristics have been evaluated using the FERMISV generator. When the three lepton flavours are combined, a total of $\sim 0.9$ such events is expected to be selected (with an additional $\sim 0.5$ if $\mathrm{q} \overline{\mathrm{q}} \nu \bar{\nu}$ final states are included). While this is in agreement with the observation of one event, the process $\mathrm{e}^{+} \mathrm{e}^{-} \rightarrow 1^{+} 1^{-} \nu \bar{\nu}$ tends to produce events which cluster toward the lowest $m$ and $p_{t}$ values. The probability for such an event, once selected, to lie beyond $3 \mathrm{GeV} / c^{2}$ in mass and $20 \mathrm{GeV} / c$ in transverse momentum is found to be $0.3 \%$. The probability for an event to show up in as unlikely a configuration (maybe more unlikely in $m$ and less in $p_{t}$, or the reverse) is $2 \%$. On the other hand, if this event were to be interpreted as coming from the process $\mathrm{e}^{+} \mathrm{e}^{-} \rightarrow \mathrm{he}^{+} \mathrm{e}^{-}$, the value of the mass of the $\mathrm{e}^{+} \mathrm{e}^{-}$pair would be unexpectedly low, with a probability of only $1 \%$.

Calculated with respect to all $\mathrm{hZ}^{*}$ final states, the combined efficiency of the searches for acoplanar lepton pairs, for monojets and for acoplanar jets increases smoothly from $10 \%$ for a massless Higgs boson to $50 \%$ for $m_{\mathrm{h}}=45 \mathrm{GeV} / c^{2}$ and then decreases slowly to $40 \%$ for $m_{\mathrm{h}}=70 \mathrm{GeV} / c^{2}$. The $95 \% \mathrm{CL}$ upper limit on the ratio $\xi^{2}$ of the production crosssection of such an invisible Higgs boson to the corresponding cross-section for a minimal standard model Higgs boson is presented in Fig. 1, as a function of $m_{\mathrm{h}}$. The cusp at $m_{\mathrm{h}}=61.3 \mathrm{GeV} / c^{2}$ is due to the candidate event, with the mass information taken into account according to the prescription of Ref. 12. For $m_{\mathbf{h}}=0, \xi^{2}$ is constrained to be smaller than $2.310^{-3}$. For $\xi^{2}=1$, the lower limit on $m_{\mathrm{h}}$ is $65 \mathrm{GeV} / c^{2}$. This seemingly large value is due to the fact that, in contrast to the MSM Higgs boson case, the hadronic $\mathrm{Z}^{*}$ decays can be used in the search for an invisible Higgs boson.

If only a fraction $f_{i n v}$ of the Higgs boson decays is invisible, the upper limit $\xi_{\text {mix }}^{2}$ on $\xi^{2}$ lies between $\xi_{i n v}^{2}$, the limit obtained here for a purely invisible Higgs boson, and $\xi^{2}{ }_{v i s}$, the limit obtained in Section 2 or in Section 3, depending on whether h does not or does decay into $\mathrm{AA}$. The actual value is given by $1 / \xi^{2}{ }_{m i x}=f_{i n v} / \xi^{2}{ }_{i n v}+\left(1-f_{\text {inv }}\right) / \xi^{2}{ }_{v i s}$. 


\section{Implications in the MSSM}

The Higgs sector of the MSSM is highly constrained [1]. At tree level, all masses and all couplings depend on only two parameters which can conveniently be chosen as $m_{\mathrm{h}}$ and $m_{\mathrm{A}}$. However, because of the large value of the top quark mass $m_{\mathrm{t}}$, one-loop radiative corrections to the $\mathrm{CP}$-even squared mass matrix must be taken into account [13]. Assuming for simplicity that the scalar partners $\tilde{t}$ of the top quark are mass degenerate, these corrections can be parametrized as $\varepsilon_{0} / \sin ^{2} \beta$, with [14]

$$
\varepsilon_{0}=\frac{3 g^{2}}{8 \pi^{2}} \frac{m_{\mathrm{t}}^{4}}{m_{\mathrm{W}}{ }^{2}} \log \frac{m_{\tilde{t}_{\mathrm{t}}^{2}}}{m_{\mathrm{t}}{ }^{2}} .
$$

For the "typical" set of values $m_{\mathrm{t}}=140 \mathrm{GeV} / c^{2}$ and $m_{\tilde{\mathrm{t}}}=1 \mathrm{TeV} / c^{2}, \varepsilon_{0}=\left(60 \mathrm{GeV} / c^{2}\right)^{2}$. As in any two-doublet model, the ZZh and ZhA couplings are proportional to $\sin (\beta-\alpha)$ and to $\cos (\beta-\alpha)$, respectively. Therefore, searches for the bremsstrahlung process $\mathrm{e}^{+} \mathrm{e}^{-} \rightarrow \mathrm{hZ}^{*}$ and for the associated production process $\mathrm{e}^{+} \mathrm{e}^{-} \rightarrow \mathrm{hA}$ are complementary. In the rest of this section, the usual assumption that $\tan \beta$ is larger than unity is made, as suggested by the large ratio of the top to the bottom-quark masses.

Let it first be assumed that decay modes into supersymmetric particles are unavailable, in particular invisible decay modes. The $\xi^{2}$ limits obtained in Sections 2 and 3 can be used to reduce the allowed domain in the $\left(m_{\mathbf{h}}, m_{\mathrm{A}}\right)$ plane, as shown in Fig. 3a for the typical set of parameters. The searches for signals of associated production of $h$ and $A$ in the data sample collected by ALEPH in 1989 and 1990 have been described in Ref. 3, and updated as reported in Ref. 15 to include the data accumulated in 1991 . The resulting exclusion domain is shown in Fig. $3 \mathrm{~b}$ for the typical set of parameters. Since the associated production process is already kinematically limited, in contrast to the bremsstrahlung process, there remains little to gain from the analysis of more data. Combining the two sets of results, the domain shown in Fig. 3c is excluded, which allows mass lower limits of 44.5 and $45 \mathrm{GeV} / c^{2}$ to be derived for $m_{\mathrm{h}}$ and $m_{\mathrm{A}}$, respectively.

These limits are however valid only for the typical choice of parameters, $m_{\mathrm{t}}=$ $140 \mathrm{GeV} / c^{2}$ and $m_{\tilde{\mathrm{t}}}=1 \mathrm{TeV} / c^{2}$. The effect of varying these parameters is demonstrated in Figs. 3d and 3e, obtained with $m_{\mathrm{t}}=100$ and $180 \mathrm{GeV} / \mathrm{c}^{2}$, respectively (and keeping the $m_{\tilde{\mathrm{t}}} / m_{\mathrm{t}}$ ratio fixed). While the lower bound on $m_{\mathrm{h}}$ is hardly affected, the lower bound on $m_{\mathrm{A}}$ is degraded for large $m_{\mathrm{t}}$ values. The set of points in the $\left(m_{\mathrm{h}}, m_{\mathrm{A}}\right)$ plane which is excluded irrespective of the values taken by $m_{\mathrm{t}}$ and by $m_{\tilde{\mathrm{t}}}>m_{\mathbf{t}}$ is limited by curve (A) in Fig. 3f. This boundary comes: $i$ ) on the left side from the negative search for $\mathrm{Z} \rightarrow \mathrm{hZ}^{*}$, interpreted in the case of a very light top quark $\left(\varepsilon_{0}=0\right)$; ii) on the right side from the theory, in the case of a very heavy top quark $\left(\varepsilon_{0} \rightarrow \infty\right)$; iii) on the low side from the negative search for $\mathrm{Z} \rightarrow \mathrm{hA}$, along the kinematic limit for this process. It can be seen that, in all cases, $m_{\mathrm{h}}$ and $m_{\mathrm{A}}$ have to exceed 44 and $21 \mathrm{GeV} / c^{2}$, respectively. The particular cases of long-lived Higgs bosons with masses smaller than $2 m_{\mu}$ are either unphysical in the case of h, as can be seen in Fig. 3, or already excluded by dedicated searches [8] in the case of $\mathrm{A}$. 
Given the present limits on supersymmetric particles [3], Higgs boson decays into such particles are not relevant in the mass range considered here, except possibly for the invisible modes $(\mathrm{h}$ or $\mathrm{A}) \rightarrow \chi \chi$. Indeed, LEP data exclude $m_{\chi} \lesssim 15 \mathrm{GeV} / c^{2}$ for $\tan \beta \gtrsim 2[3]$, while the limits obtained for the gluino mass in $\mathrm{p} \overline{\mathrm{p}}$ collisions [16] can be used in the MSSM framework to extend the $15 \mathrm{GeV} / c^{2}$ lower limit on $m_{\chi}$ into the $1<\tan \beta<2$ region. These constraints do not forbid e.g. $\mathrm{h} \rightarrow \chi \chi$ if $m_{\mathrm{h}}=50 \mathrm{GeV} / c^{2}$ and $m_{\chi}=20 \mathrm{GeV} / c^{2}$. In addition, there remains a so-called "light gluino window" in the mass region 3 to $4 \mathrm{GeV} / \mathrm{c}^{2}$ which is not fully excluded experimentally. As a consequence, the possibility of a very light $\chi$, with $\tan \beta$ close to unity, should not be overlooked. This means that invisible decay modes should be considered for any Higgs boson masses, especially in view of their potentially large branching ratios $[6]$.

If $\mathrm{h}$ can decay invisibly, the domain shown in Fig. 3a, excluded for the typical set of parameters by the search for $\mathrm{e}^{+} \mathrm{e}^{-} \rightarrow \mathrm{hZ}^{*}$, can only become larger since the limits obtained on $\xi^{2}$ are tighter for an invisible than for a visible Higgs boson. On the other hand, the searches for $\mathrm{e}^{+} \mathrm{e}^{-} \rightarrow \mathrm{hA}$ described in Ref. 3 become inefficient, and the results displayed in Fig. $3 \mathrm{~b}$ are no longer valid. Constraints resulting from the $\mathrm{Z}$ width measurement can however be used in this case too, as explained in Ref. 3, and the practical result is that the window at low $h$ and $A$ masses which is visible in Fig. 3a becomes closed. The set of points which is excluded, whatever the invisible fractions of h and A decays, when $m_{\mathrm{t}}$ and $m_{\tilde{\mathrm{t}}}>m_{\mathrm{t}}$ are allowed to vary arbitrarily, is limited by curve (B) in Fig. 3f.

\section{Conclusions}

Searches have been performed for signals of the production of a non-minimal CP-even Higgs boson $h$ in the bremsstrahlung process $\mathrm{e}^{+} \mathrm{e}^{-} \rightarrow \mathrm{hZ}^{*}$. Only one candidate event was found, an acoplanar $\mathrm{e}^{+} \mathrm{e}^{-}$pair which may be interpreted at the few percent probability level as coming from the process $\mathrm{e}^{+} \mathrm{e}^{-} \rightarrow \mathrm{e}^{+} \mathrm{e}^{-} \nu \bar{\nu}$.

Limits on $\xi^{2}$, the ratio of the $\mathrm{e}^{+} \mathrm{e}^{-} \rightarrow \mathrm{hZ} \mathrm{Z}^{*}$ production cross-section to its MSM equivalent, have been derived as a function of $m_{\mathrm{h}}$ in the cases where h decays like an MSM Higgs boson, with possibly modified branching ratios, into a pair of $\mathrm{A}$ bosons, or into invisible final states. For a purely invisible Higgs boson produced with the same cross-section as in the MSM, the mass lower limit is $65 \mathrm{GeV} / \mathrm{c}^{2}$.

Together with previous ALEPH results on the associated production process $\mathrm{e}^{+} \mathrm{e}^{-} \rightarrow \mathrm{hA}$, these limits exclude a domain in the $\left(m_{\mathrm{h}}, m_{\mathrm{A}}\right)$ plane of the MSSM, the area of which depends on the size of the radiative corrections to the CP-even squared mass matrix and on the availability of supersymmetric decay modes. If such decay modes are forbidden, mass lower limits of 44.5 and $45 \mathrm{GeV} / c^{2}$ result for the $\mathrm{h}$ and A bosons, respectively, assuming $m_{\mathrm{t}}=140 \mathrm{GeV} / c^{2}, m_{\tilde{\mathrm{t}}}=1 \mathrm{TeV} / c^{2}$ and $\tan \beta>1$. If $m_{\mathrm{t}}$ and $m_{\tilde{\mathrm{t}}}>m_{\mathrm{t}}$ are allowed to vary arbitrarily, these limits become 44 and $21 \mathrm{GeV} / c^{2}$, respectively.

These results improve on those obtained in previous analyses [3,17], and are the first ones reported in the case of invisible Higgs decays. 


\section{Acknowledgements}

We wish to thank our colleagues from the accelerator divisions for the successful operation of LEP. We are indebted to the engineers and technicians in all our institutions for their contribution to the good performance of ALEPH. Those of us from non-member countries thank CERN for its hospitality.

\section{References}

1. J.F. Gunion, H.E. Haber, G. L. Kane and S. Dawson, "The Higgs Hunter's Guide" (Addison Wesley, 1990).

2. M. Veltman, Nucl. Phys. B 123 (1977) 89.

3. ALEPH Coll., D. Decamp et al., Phys. Rep. 216 (1992) 253.

4. ALEPH Coll., "Search for the standard model Higgs boson", CERN-PPE/93-110, July 1993; to be published in Physics Letters B.

5. A.S. Joshipura and J.W.F. Valle, Nucl. Phys. B 397 (1993) 105.

6. A. Djouadi, J. Kalinowski and P.M. Zerwas, Z. Phys. C 57 (1993) 569.

7. ALEPH Coll. D. Decamp et al., Phys. Lett. B 265 (1991) 475.

8. ALEPH Coll., D. Buskulic et al., Phys. Lett. B 285 (1992) 309.

9. ALEPH Coll. D. Decamp et al., Nucl. Inst. and Methods A 294 (1990) 121.

10. S. Dagoret, Thesis (Université Paris-Sud, May 1991), LAL 91-12.

11. J. Hilgart, R. Kleiss and F. Le Diberder, Comp. Phys. Comm. 75 (1993) 191.

12. J.-F. Grivaz and F. Le Diberder, "On the determination of a mass lower limit for the Higgs boson in the presence of candidate events", LAL 92-23, April 1992, and erratum LAL 92-45, August 1992; to be published in Nucl. Inst. and Methods.

13. J. Ellis, G. Ridolfi and F. Zwirner, Phys. Lett. B 262 (1991) 477, and earlier references therein.

14. R. Barbieri and M. Frigeni, Phys. Lett. B 258 (1991) 395.

15. ALEPH Coll., "Improved mass limits for the neutral Higgs bosons of the MSSM", Contributed paper to the XXVI ${ }^{\text {th }}$ Int. Conf. on High Energy Physics, 6-12 August 1992, Dallas.

16. CDF Coll., F. Abe et al., Phys. Rev. Lett. 69 (1992) 3439.

17. L3 Coll., O. Adriani et al., Z. Phys. C 57 (1993) 355. 


\section{Figure Captions}

1. As a function of $m_{\mathrm{h}}$, the $95 \% \mathrm{CL}$ upper limit on $\xi^{2}$, the ratio of the production crosssection of a non-minimal CP-even Higgs boson $h$ in the reaction $\mathrm{e}^{+} \mathrm{e}^{-} \rightarrow \mathrm{hZ}^{*}$ to the corresponding cross-section for a MSM Higgs boson.

Curve (A) applies if h decays into the same final states as an MSM Higgs boson, with modified branching ratios; the discontinuities correspond to thresholds for various $h$ decay modes $\left(\pi \pi, \mathrm{K} \overline{\mathrm{K}}, \tau^{+} \tau^{-}\right.$and $\mathrm{c} \overline{\mathrm{c}}$, and $\left.\mathrm{b} \overline{\mathrm{b}}\right)$, except for the one at $40 \mathrm{GeV} / c^{2}$ which is related to the recoil mass cut in the $\mathrm{hl}^{+} 1^{-}$channel; there is no general upper limit on $\xi^{2}$ for $m_{\mathrm{h}}<2 m_{\mu}$. If $\mathrm{h}$ decays into $\mathrm{AA}$, the limit $(\mathrm{A})$ holds if $m_{\mathrm{A}} \gtrsim 2 m_{\mathrm{b}}$; this limit is degraded (i.e translated upwards) by no more than $10 \%$ for lower A masses.

Curve (B) applies if h decays invisibly; the cusp at $61.3 \mathrm{GeV} / \mathrm{c}^{2}$ corresponds to the candidate acoplanar $\mathrm{e}^{+} \mathrm{e}^{-}$pair.

2. The selected acoplanar $\mathrm{e}^{+} \mathrm{e}^{-}$pair. The mass of the pair is $3.31 \mathrm{GeV} / c^{2}$, its transverse momentum is $20.3 \mathrm{GeV} / c$, and the mass of the invisible system recoiling against the $\mathrm{e}^{+} \mathrm{e}^{-}$ pair is $61.3 \mathrm{GeV} / c^{2}$.

3. Excluded domains in the $\left(m_{\mathrm{h}}, m_{\mathrm{A}}\right)$ plane of the MSSM:

a) from the search for $\mathrm{Z} \rightarrow \mathrm{h} \mathrm{Z}^{*}$,

b) from the search for $\mathrm{Z} \rightarrow \mathrm{hA}$, and

c) from these two searches combined,

all for the typical values $m_{\mathrm{t}}=140 \mathrm{GeV} / c^{2}$ and $m_{\tilde{\mathrm{t}}}=1 \mathrm{TeV} / c^{2}$, for $\tan \beta>1$, and assuming no invisible Higgs boson decays.

The same as (c):

d) for $m_{\mathrm{t}}=100 \mathrm{GeV} / c^{2}$, and

e) for $m_{\mathrm{t}}=180 \mathrm{GeV} / c^{2}$,

both with $m_{\tilde{\mathrm{t}}} / m_{\mathrm{t}}$ as in (c).

f) Limited by curve (A), the domain excluded for any $m_{\mathrm{t}}$ or $m_{\tilde{\mathrm{t}}}>m_{\mathrm{t}}$ values, assuming no invisible Higgs boson decays; limited by curve (B), the same, now allowing for arbitrary fractions of invisible $h$ and $A$ decays. 


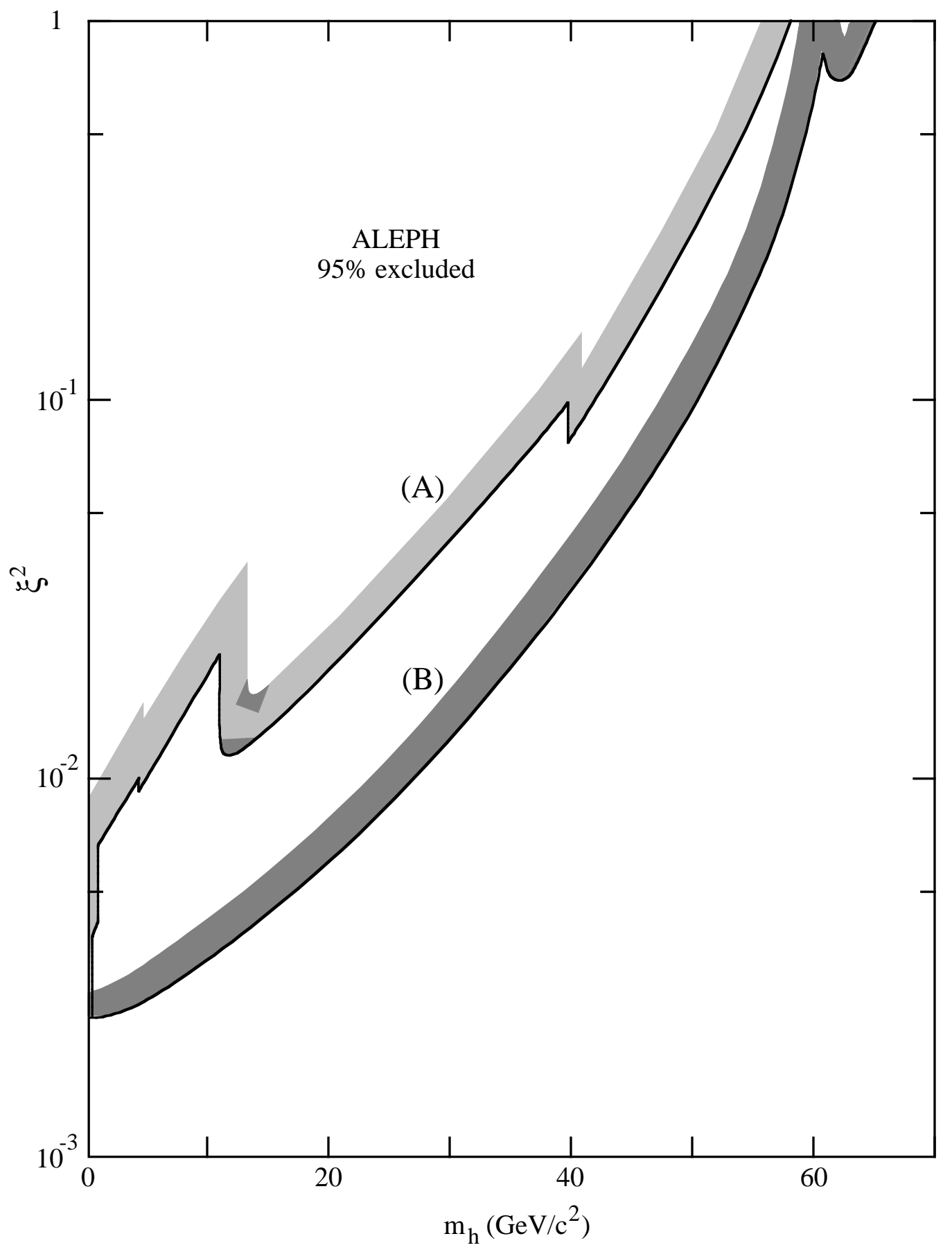

Figure 1 

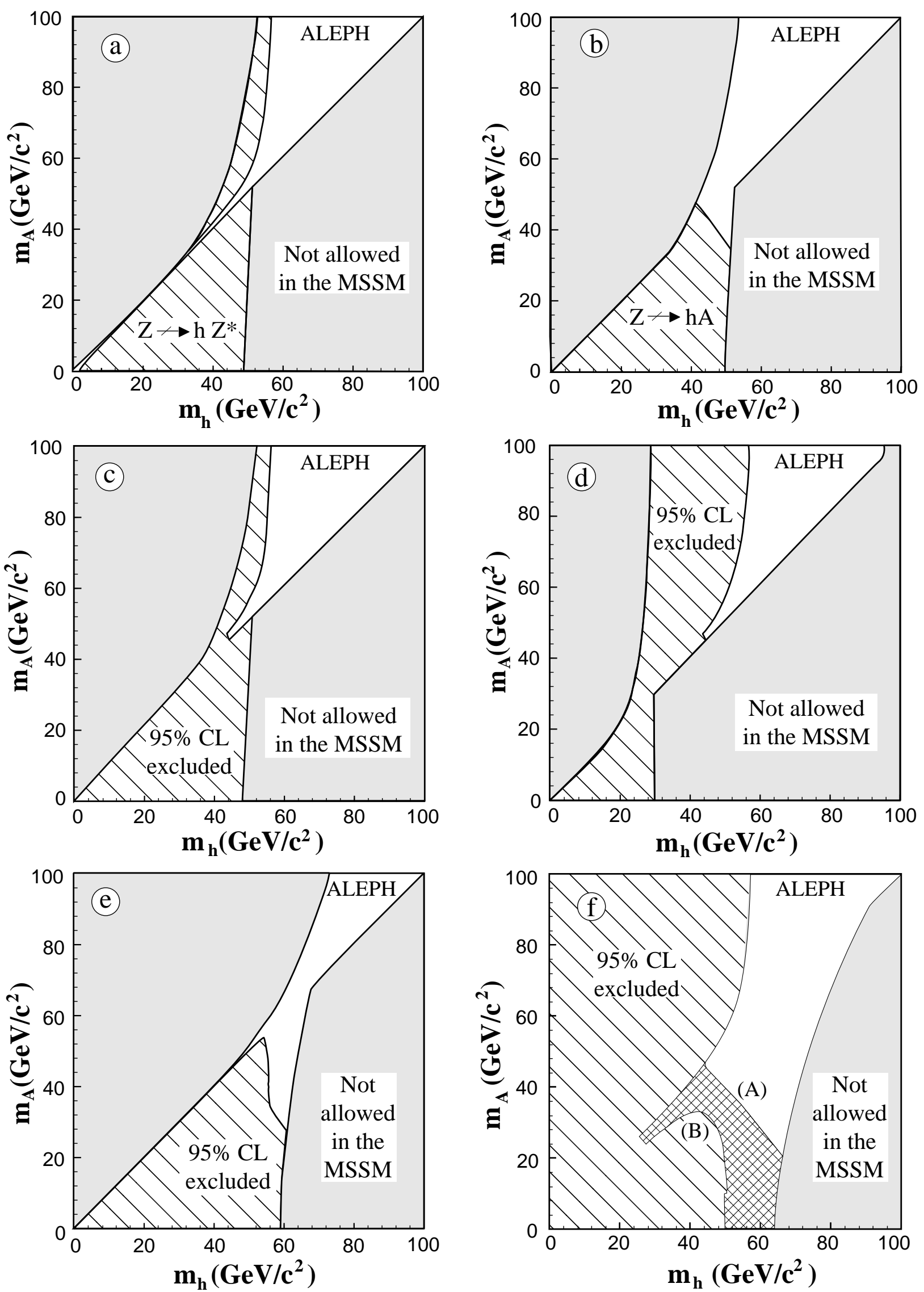

Figure 3 